\title{
A Numerical Method for Rating Thermal Performance in Spiral Heat Exchangers
}

\author{
Martin Martinez Garcia ${ }^{1} \&$ Miguel Angel Moreles ${ }^{2}$ \\ ${ }^{1}$ Departamento de Ingeniería Química, División de Ciencias Naturales y Exactas, Universidad de Guanajuato, \\ Mexico \\ ${ }^{2}$ Centro de Investigación en Matemáticas, Jalisco S/N, Valenciana, Mexico \\ Correspondence: Martin Martinez Garcia, Departamento de Ingeniería Química, División de Ciencias Naturales y \\ Exactas, Universidad de Guanajuato, Mexico. E-mail: cimba_42@hotmail.com
}

Received: April 25, 2012 Accepted: May 15, $2012 \quad$ Online Published: May 23, 2012

doi:10.5539/mas.v6n6p54 URL: http://dx.doi.org/10.5539/mas.v6n6p54

\begin{abstract}
The steady state heat transfer equations associated to two fluids in counter flow, hot and cold, in a spiral heat exchanger are considered in this study. A numerical method for solution was proposed to approximate the temperature distribution and overall heat transfer coefficients using the flow rates and the temperatures at inlets and outlets. In particular the effectiveness and correction factor were computed as performance parameters and develop a tool for design. The method is tested and validated on two actual spiral heat exchangers that were reported in the literature.
\end{abstract}

Keywords: spiral plate heat exchangers, compact heat exchangers, heat transfer

\section{Introduction}

A heat exchanger is a device in which energy is transferred from one fluid to another across a solid surface. This work is concerned with a spiral plate heat exchanger (SPHE), and it consists of two plates wound round each other maintaining a constant distance in spiral to form two concentric channels. By winding a conduit into spiral form, the efficiency of the equipment increases significantly in comparison to that of a straight structure (Wilhelmsson, 2005). These geometric characteristics and thermal efficiencies of a SPHE, makes it an excellent choice for various applications, especially for cooling viscous fluids and slurries.

In a SPHE, the hot fluid enters the central part of the team and flows outwardly (Figure 1), while the cold fluid is counter flow. In practice the flow channels for both fluids have the same settings, hence heat transfer and pressure drop properties are equal. What may differ are the dimensions of flow passages, leading to different conditions for analysis and equipment design. The purpose of current research is to determine the effects of relevant parameters in this design. A common approach is by numerical modeling the dynamic behavior of SPHE. The first step is to derive differential equations associated to the heat transfer problem, and then appropriate numerical methods are applied to simulate the phenomenon. This virtual lab becomes a powerful tool for design.

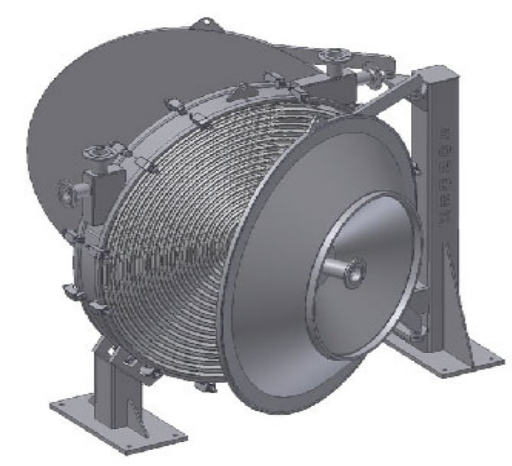

Figure 1. Spiral heat exchanger with end-cap removed (Giving access to one spiral channel) 
The differential equations models depend on the underlying physical assumptions, as well as the numerical techniques of solution. Bounopane and Troupe (1970) solved a differential model iteratively by a Runge-Kutta technique for an exchanger with six double turns for both equal and non-equal counter flows. Cieslinski and Bes (1992) used Hermite polynomials to develop a solution for a double spiral with entry and exit on opposite sides of the periphery, as in Figure 2. Zaleski and Lachowski (1984) used the method of characteristics to solve their set of differential equations representing 20 double turns of an exchanger designed to cool sulfuric acid with water. Using the Laplace transform, they developed a solution for the thermal behavior during start up, taking into account the heat capacity of the walls as well as that of the fluids. Choudhury et al. (1985) carried out a numerical study on the effects of the geometric structure of SPHE in heat flow. A similar work was performed by Martin (1992). Based on some simplifying assumptions, Morimoto and Hotta (1988) developed a more analytical study. For a more engineering prospective could saw in the works of Bes and Roetzel (1992) and Strelow (2000). Finally a more sophisticated numerical study using computational fluid dynamics (CFD) was presented in Egner and Burmeister (2005). Some experiments and empirical data were reported in Minton (1970), Holger (1992), Strenger (1990) and Saravanan (2008).

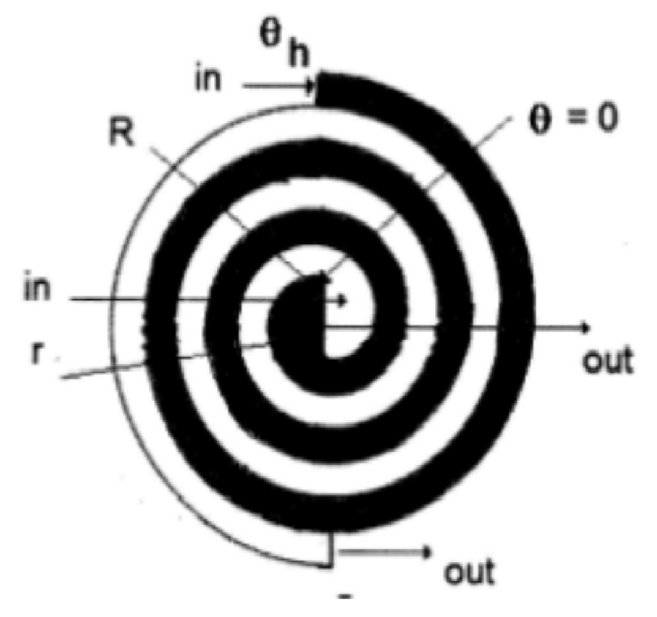

Figure 2. Flow configuration for the Spiral Heat Exchanger

It is apparent that there is no numerical model that works in general. This study is a modest step in that direction and it is considered the heat transfer equations for temperature of hot and cold fluids of Morimoto and Hotta (1988). This model includes the main parameters and coefficients for design of SPHE. This study focused on the steady state equations, the system is delayed-advanced type (Hupkes \& Augeraud-Veron, 2011). The direct application of classical numerical techniques is not plausible; hence we propose a somewhat general numerical method to compute temperature profiles. Consequently, the main coefficients for thermal performance are computed.

The outline is as follows Section 2 presented the heat transfer equations of Morimoto and Hotta (1988) and reviews the main parameters of SPHE design, namely, effectiveness $(\varepsilon)$ and correction factor for the logarithmic mean temperature difference (F). An iterative numerical method is developed in Section 3 to compute temperature profiles for the steady state equations. In Section 4 the design approach introduced in this work is illustrated using two study cases reported in Minton (1970) and Saravanan (2008). In terms of $\varepsilon$ and F, we rate several configurations of SPHE using different values for numbers of turns, number of thermal units (NTU), and capacity ratio $\mathrm{C}$. A case is made for this numerical rating as a design tool. In the last section conclusions are drawn and future research directions are outlined.

\section{A Model for Thermal Performance and Design Parameters for a SPHE}

A spiral heat exchanger is characterized by having a pair of rolled plates and maintaining a constant spacing between them forming concentric flow channels. Except for the center and periphery, heat is exchanged through the bilateral sides of the channels. It is assumed that the cross-flow section is rectangular with a constant aspect ratio. This geometric configuration of the exchanger increases efficiency significantly. Moreover, plate spacers improve turbulence while velocities increase thus avoiding fouling problems.

Derivation of the fundamental equations of spiral heat exchanger are based on the following assumptions: (1) fluid temperature is uniform in the channel cross section perpendicular to the flow direction, (2) conduction in 
the direction of flow can be neglected, (3) heat-transfer coefficient is independent of the fluid temperature and curvature of the channel, (4) uniform physical properties of the fluid in the device.

Due to the direction of the centrifugal force and the location of cold and hot fluid, two coefficients of individual heat transfer are necessary for the analysis. We are led to the mathematical model that follows.

Cold fluid:

$0 \leq \theta \leq \pi$

$$
r(\theta) \frac{\partial t(\theta, \tau)}{\partial t}=\frac{w}{\rho \delta b} \frac{\partial t(\theta, \tau)}{\partial \theta}+\frac{h_{c}}{c \rho \delta}\left\{r(\theta)+\frac{\delta}{2}\right\}\{T(\theta+2 \pi, \tau)-t(\theta, \tau)\}
$$

$\pi \leq \theta \leq\left(\theta_{h}-2 \pi\right)$,

$$
\begin{aligned}
r(\theta) \frac{\partial t(\theta, \tau)}{\partial \tau}= & \frac{w}{\rho \delta b} \frac{\partial t(\theta, \tau)}{\partial \theta}+\frac{h_{c}}{c \rho \delta}\left\{r(\theta)+\frac{\delta}{2}\right\}\{T(\theta+2 \pi, \tau)-t(\theta, \tau)\} \\
& +\frac{h_{h}}{c \rho \delta}\left\{r(\theta)-\frac{\theta}{2}\right\}\{T(\theta, \tau)-t(\theta, \tau)\}
\end{aligned}
$$

$\left(\theta_{h}-2 \pi\right) \leq \theta \leq \theta_{c}$,

$$
r(\theta) \frac{\partial t(\theta, \tau)}{\partial t}=\frac{w}{\rho \delta b} \frac{\partial t(\theta, \tau)}{\partial \theta}+\frac{h_{h}}{c \rho \delta}\left\{r(\theta)-\frac{\delta}{2}\right\}\{T(\theta, \tau)-t(\theta, \tau)\}
$$

Hot fluid:

$\pi \leq \theta \leq 2 \pi$

$$
R(\theta) \frac{\partial T(\theta, \tau)}{\partial \tau}=\frac{W}{\rho \delta b} \frac{\partial T(\theta, \tau)}{\partial \theta}+\frac{h_{h}}{C \rho \delta}\left\{R(\theta)+\frac{\delta}{2}\right\}\{t(\theta, \tau)-T(\theta, \tau)\}
$$

$2 \pi \leq \theta \leq \theta_{c}$

$$
R(\theta) \frac{\partial T(\theta, \tau)}{\partial \tau}=\frac{W}{\rho \delta b} \frac{\partial T(\theta, \tau)}{\partial \theta}+\frac{h_{h}}{C \rho \delta}\left\{R(\theta)+\frac{\delta}{2}\right\}\{t(\theta, \tau)-T(\theta, \tau)\}
$$

$2 \pi \leq \theta \leq \theta_{c}$

$$
\begin{aligned}
R(\theta) \frac{\partial T(\theta, \tau)}{\partial \tau}= & \frac{W}{\rho \delta b} \frac{\partial T(\theta, \tau)}{\partial \theta}+\frac{h_{h}}{C \rho \delta}\left\{R(\theta)-\frac{\delta}{2}\right\}\{t(\theta-2 \pi, \tau)-T(\theta, \tau)\}+ \\
& \frac{h_{c}}{C \rho \delta}\left\{R(\theta)+\frac{\delta}{2}\right\}\{t(\theta, \tau)-T(\theta, \tau)\}
\end{aligned}
$$

Figure 3 shows the channel range where each equation holds, as well as typical temperature distribution in the apparatus.

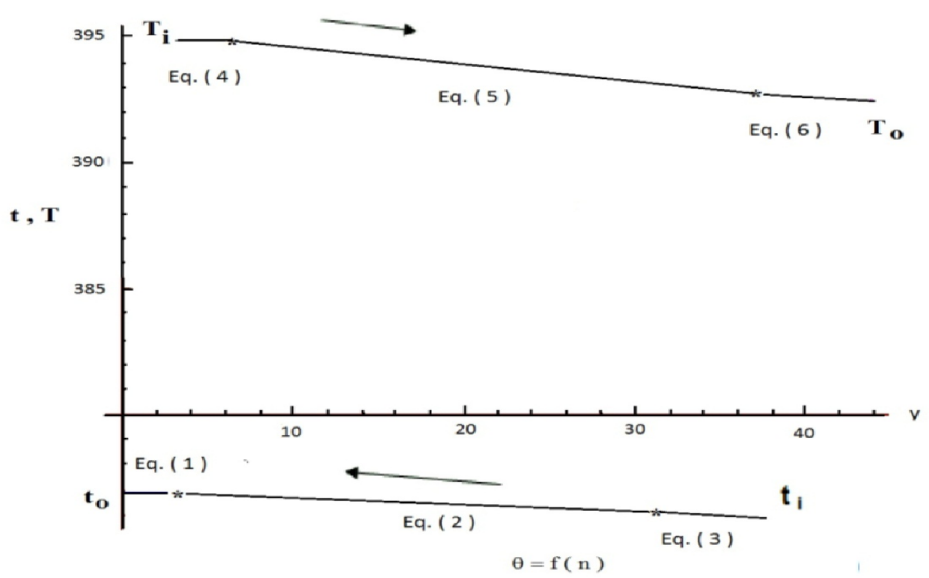

Figure 3. Temperature profiles and equations for each section 
For design we assume that steady state has been reached. Consequently we shall consider the system of ordinary differential equations of delayed-advanced type:

Cold fluid:

$0 \leq \theta \leq \pi$

$$
\frac{d t}{d \theta}=\frac{b h_{c}}{w c}\left\{r(\theta)+\frac{\delta}{2}\right\}\{t(\theta, \tau)-T(\theta+2 \pi, \tau)\}
$$

$\pi \leq \theta \leq\left(\theta_{h}-2 \pi\right)$

$$
\frac{d t}{d \theta}=\frac{b h_{c}}{w c}\left\{r(\theta)+\frac{\delta}{2}\right\}\{t(\theta)-T(\theta+2 \pi)\}+\frac{b h_{c}}{w c}\left\{r(\theta)-\frac{\delta}{2}\right\}\{t(\theta)-T(\theta)\}
$$

$\left(\theta_{h}-2 \pi\right) \leq \theta \leq \theta_{c}$

$$
\frac{d t}{d \theta}=\frac{b h_{h}}{w c}\left\{r(\theta)-\frac{\delta}{2}\right\}\{t(\theta)-T(\theta)\}
$$

Hot fluid:

$\pi \leq \theta \leq 2 \pi$;

$$
\frac{d T}{d \theta}=\frac{b h_{h}}{W C}\left\{R(\theta)+\frac{\delta}{2}\right\}\{T(\theta)-t(\theta)\}
$$

$2 \pi \leq \theta \leq \theta_{c}$

$$
\frac{d T}{d \theta}=\frac{b h_{h}}{W C}\left\{R(\theta)-\frac{\delta}{2}\right\}\{T(\theta)-t(\theta-2 \pi)\}+\frac{b h_{h}}{W C}\left\{R(\theta)+\frac{\delta}{2}\right\}\{T(\theta)-t(\theta)\}
$$

$\theta_{c} \leq \theta \leq \theta_{h}$

$$
\frac{d T}{d \theta}=\frac{b h_{c}}{W C}\left\{R(\theta)-\frac{\delta}{2}\right\}\{T(\theta)-t(\theta-2 \pi)\}
$$

Thermal performance of a SPHE may now determine in three steps:

1) Input fluid properties and compute geometric parameters,

2) Solve steady state equations to obtain temperature profiles,

3) In terms of temperature profiles, compute thermal performance parameters.

In regards to step 1) we refer to the double spiral shown in Figure 4, the spiral is made up of a series of half-cylinders of discretely varying size for ease in fabrication and differs thereby only slightly from a true Archimedean spiral with a constantly changing radius of curvature. The number of turns of the channel through for fluid flow from periphery to the core is denoted by $n$, which is also the number of turns of the exiting stream. To compute the area of heat transfer we may consider that heat transfer from the periphery to the surroundings and through the walls of the core as negligible. Consequently, if $\mathrm{H}$ is the plate's width and $r_{\text {avg }}$ the average radius, the area for heat transfer in the inner channels is $4 \pi \mathrm{H}(\mathrm{n}-1 / 2)$ ravg and $4 \pi$ Hnravg for the device in Figure 2. Computation of the remaining geometric parameters is straightforward.

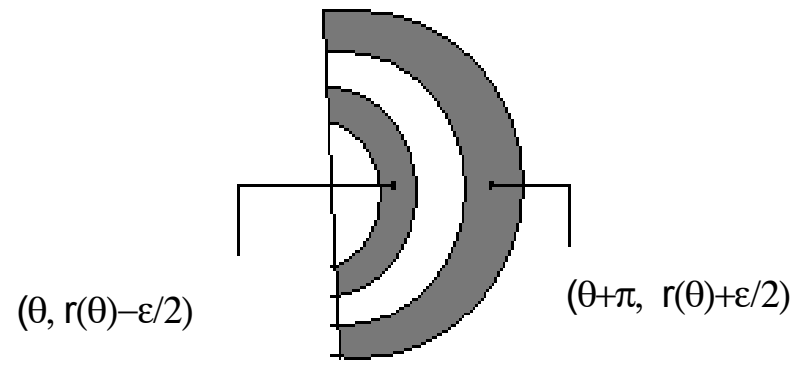

Figure 4. Basis for the model geometry channels spiral plate

For Step 2 a method of solution is proposed in the next Section.

From the temperature profiles obtained in Step 2 we obtain the outlet temperatures $t_{0}$ and $T_{0}$ In terms of these outlet temperatures SPHE performance parameters are computed, namely, the correction factor for the 
logarithmic mean temperature difference $\mathrm{F}$ and the thermal effectiveness $\varepsilon$. Let us review this computation to conclude Step 3.

In applications the most common expressions for the correction factor F are due to Holger (1992) and Bes and Roetzel (1992), which are respectively

$$
\begin{gathered}
F_{H M}=\frac{n}{N} \tanh \frac{N}{n} \\
\left.F_{B}=\ln 1+C N^{2}\right) / C N^{2} \\
C N=2\left[\left(N T U_{c} / N T U_{h}\right)^{1 / 2}\right]\left(\pi A_{c} A\right)^{1 / 2}
\end{gathered}
$$

When there is no confusion, we shall denote by F the correction factor. In terms of F, effectiveness is computed by:

$$
\varepsilon=\frac{1-e^{(C-1) N^{*} F}}{1-C^{*} e^{(C-1) N^{*} F}}
$$

$F$ is a measure of the performance as compared to a true countercurrent exchanger. In terms of the number of thermal transfer units:

$$
N=U A / w c
$$

Also recall that the basic equation for heat exchanger associated to the thermal load $\mathrm{Q}$, is $Q=U A F(\Delta T)_{l m}$. The overall coefficient of heat transfer $U$ is estimated from operating data and the geometric plate heat exchanger coil.

It is apparent that a robust numerical solution of equations (7)-(12) becomes a tool for design by analyzing correlations of the effectiveness and correction factor with respect to number of turns, thermal transfer units, etc.

\section{A Numerical Method for Solution}

As mentioned above, the system of steady state equations (7)-(12) is of delayed-advanced type. We propose an iterative scheme for solution.

In engineering design, a working condition for a SPHE is $\Delta \mathrm{T}=\left|\mathrm{T}_{\mathrm{o}}-\mathrm{t}_{\mathrm{o}}\right|>10^{\circ} \mathrm{C}$. This will serve as the stopping criterion.

Let k=0, and initialize $t^{0}(\theta)=t_{i} T^{0}(\theta)=T_{i} \cdot \Delta T=T_{i}-t_{i}$. The idea is to solve from right to left for the for the hot fluid, and from left to right for the cold fluid. In both cases, updated temperature is used. Note the superscripts in the temperatures below.

For a function $\mathrm{f}(\mathrm{x})$ we denote by $f\left(x_{+}\right), f\left(x_{-}\right)$the limits on the right and left respectively.

The algorithm is as follows:

While $\Delta T>10$ do

Begin (while)

$T_{o}=T^{k}\left(\theta_{h}\right), t_{o}=t^{k}(0)$,

In the given intervals solve the corresponding differential equations

$(\theta-2 \pi) \leq \theta \leq \theta_{c}$,

$$
\begin{gathered}
\frac{d t^{k+1}}{d \theta}=\frac{b h_{h}}{w c}\left\{r(\theta)-\frac{\delta}{2}\right\}\left\{t^{k+1}(\theta)-T^{k}(\theta)\right\} ; \\
t^{k+1}\left(\theta_{h}\right)=t_{i} .
\end{gathered}
$$

$\pi \leq \theta \leq(\theta-2 \pi)$,

$$
\frac{d t^{k+1}}{d \theta}=\frac{b h_{c}}{w c}\left\{r(\theta)+\frac{\delta}{2}\right\}\left\{t^{k+1}(\theta)-T^{k}(\theta+2 \pi)\right\}+\frac{b h_{c}}{w c}\left\{r(\theta)-\frac{\delta}{2}\right\}\left\{t^{k+1}(\theta)-T^{k}(\theta)\right\}
$$




$$
t^{k+1}\left(\theta_{h}-\pi\right)=t^{k+1}\left(\left(\theta_{h}-\pi\right)_{+}\right) .
$$

$0 \leq \theta \leq \pi$,

$$
\begin{gathered}
\frac{d t^{k+1}}{d \theta}=\frac{b h_{c}}{w c}\left\{r(\theta)+\frac{\delta}{2}\right\}\left\{t^{k+1}(\theta, \tau)-T^{k}(\theta+2 \pi, \tau)\right\}, \\
t^{k+1}(\pi)=t^{k+1}\left(\pi_{+}\right) .
\end{gathered}
$$

EscucharLeer fonéticamente

$\pi \leq \theta \leq 2 \pi$,

$$
\begin{gathered}
\frac{d T^{k+1}}{d \theta}=\frac{b h_{h}}{W C}\left\{R(\theta)+\frac{\delta}{2}\right\}\left\{T^{k+1}(\theta)-t^{k+1}(\theta)\right\}, \\
T^{k+1}(\pi)=T_{i} .
\end{gathered}
$$

$2 \pi \leq \theta \leq \theta_{c}$

$$
\begin{gathered}
\frac{d T^{k+1}}{d \theta}=\frac{b h_{h}}{W C}\left\{R(\theta)-\frac{\delta}{2}\right\}\left\{T^{k+1}(\theta)-t^{k+1}(\theta-2 \pi)\right\}+\frac{b h_{h}}{W C}\left\{R(\theta)+\frac{\delta}{2}\right\}\left\{T^{k+1}(\theta)-t^{k+1}(\theta)\right\}, \\
T^{k+1}(2 \pi)=T^{k+1}\left(2 \pi_{-}\right) .
\end{gathered}
$$

$\theta_{c} \leq \theta \leq \theta_{h}$,

End (while)

$$
\begin{gathered}
\frac{d T^{k+1}}{d \theta}=\frac{b h_{c}}{W C}\left\{R(\theta)-\frac{\delta}{2}\right\}\left\{T^{k+1}(\theta)-t^{k+1}(\theta-2 \pi)\right\}, \\
T^{k+1}\left(\theta_{h}\right)=T^{k+1}\left(\left(\theta_{h}\right)_{-}\right) . \\
\Delta T=\left|T^{k+1}\left(\theta_{h}\right)-t^{k+1}(0)\right| \\
k \leftarrow k+1
\end{gathered}
$$

For output we obtain temperature profiles and outlet temperatures.

\section{Results and Discussion}

Here we apply our thermal performance scheme to the SPHE reported by Minton (1970) and Saravanan (2008). In terms of the computed temperatures we obtain the behavior of LMTD correction factors and effectiveness in terms of various capacity rate ratios, number of turns and number of heat transfer units for the two cases. Implications for design are apparent.

First let us consider the operation conditions and design parameters for Minton's SPHE as described in Table 1. It corresponds to a SPHE with 12 turns. Form our algorithm we obtain the outlet temperatures $\mathrm{T}_{0}=420.421 \mathrm{~K}$, $t_{0}=395.133 \mathrm{~K}$. Relative errors with respect to those of Minton's are $0.71 \%$ and $0.5427 \%$. The approximation is satisfactory. 
Table 1. Case study I-Specifications streams

\begin{tabular}{ccc}
\hline item & Hot Fluid & Cold fluid \\
\hline Flow rate $(\mathrm{kg} / \mathrm{s})$ & 0.7833 & 0.7444 \\
Inlet temperature $\left({ }^{\circ} \mathrm{C}\right)$ & 200 & 60 \\
Outlet temperature $\left({ }^{\circ} \mathrm{C}\right)$ & 120 & 150.4 \\
Heat capacity $\left(\mathrm{J} / \mathrm{kg}^{\circ} \mathrm{C}\right)$ & 2973 & 2763 \\
Thermal conductivity $\left(\mathrm{W} / \mathrm{m}{ }^{\circ} \mathrm{C}\right)$ & 0.348 & 0.322 \\
Density $\left(\mathrm{kg} / \mathrm{m}^{3}\right)$ & 843 & 843 \\
Pressure drop $(\mathrm{Pa})$ & $6.89 \times 10^{-2}$ & $6.89 \times 10^{-2}$ \\
Viscosity $(\mathrm{kg} / \mathrm{m} \mathrm{s})$ & $3.35 \times 10^{-3}$ & $8.0 \times 10^{-3}$ \\
Plate spacing $(\mathrm{m})$ & $4.77 \times 10^{-3}$ & $6.27 \times 10^{-3}$ \\
Plate thickness $(\mathrm{m})$ & $3.175 \times 10^{-3}$ & $3.175 \times 10^{-3}$ \\
Initial internal diameter $(\mathrm{m})$ & 0.203 & 0.203 \\
Thermal conductivity of material & 17.3 & 17.3 \\
of construction $\left(\mathrm{W} / \mathrm{m}{ }^{\circ} \mathrm{C}\right)$ & & \\
\hline
\end{tabular}

In Figures (5)-(8) we show results for Case 1. In order to validate the outlet temperatures obtained from our algorithm we plot the Holger's correction factor of varying the ratio NTU/n. As shown in Figure 5 an accurate fit for the correction factor computed with the temperatures from the algorithm is obtained.

With our tools we may test the performance of Minton's SPHE as a result of its physical dimension, that is $\mathrm{NTU}=10$. For the given number of turns, notice that effectiveness is optimal for any value of the capacity ratio.

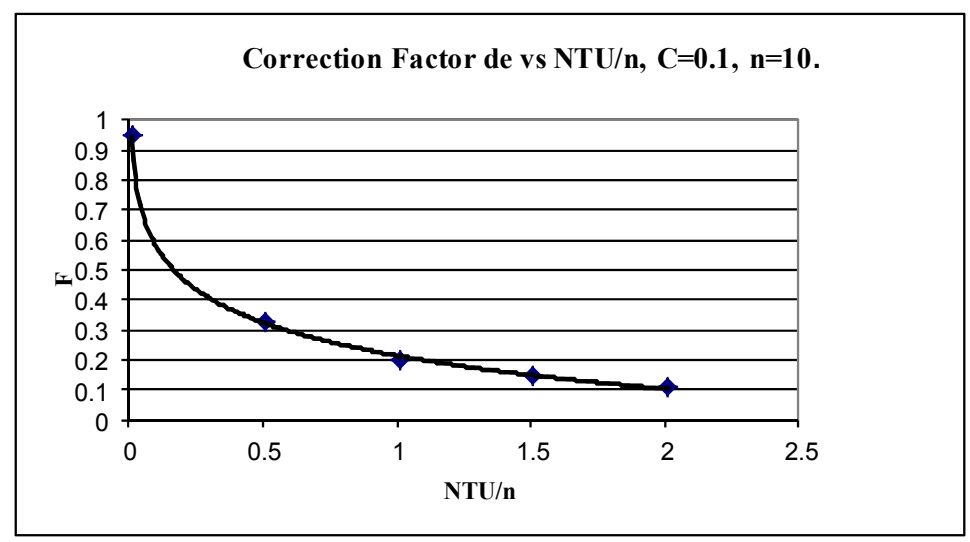

Figure 5. LMTD correction factor F vs NTU/n ratio (The points correspond to in plot are values calculated and the line are determined by Holger's Equation.)

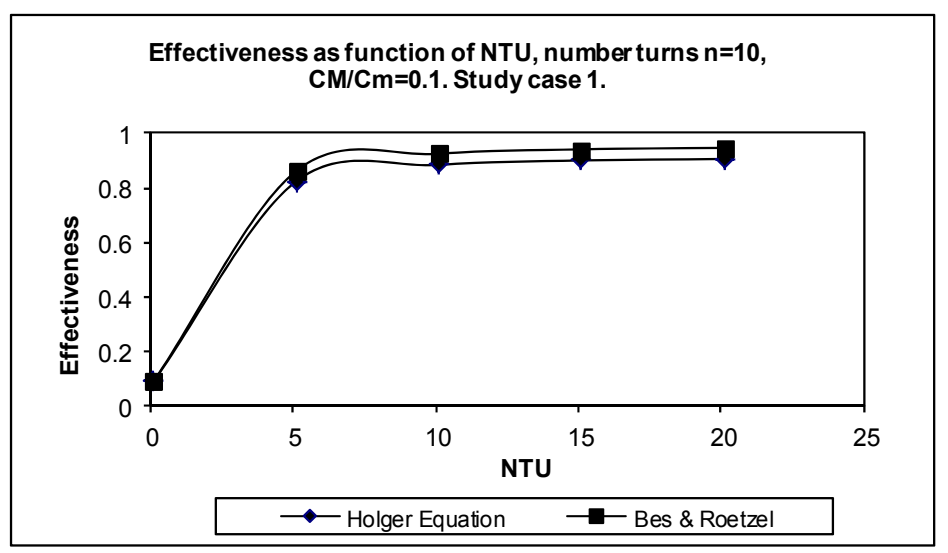

Figure 6. Efectivenness Vs NTU was calculated with Holger and Bes \&Roetzel equations with temperatures numerically were obtained, Case Study I 
In Figure 6 effectiveness with NTU is correlated. The former is computed with the equation of Holger (13). Observe that for a fixed number of turns, $n=10,10$ NTU is an optimal value. There is no improvement on effectiveness after this.

In Figures 7 and 8 we vary the number of turns and plot the values for effectiveness. It is observed that performance is not improved when the number of turns is greater than 10 .

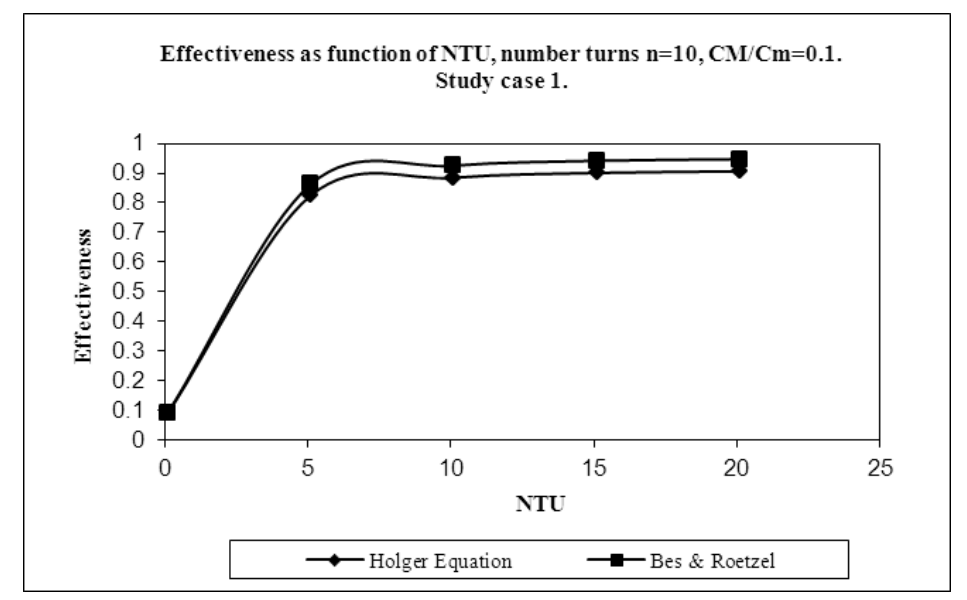

Figure 7. Effectiveness Vs NTU calculated with Holger and Bes \& Roetzel equations with temperatures numerically obtained, Case Study I

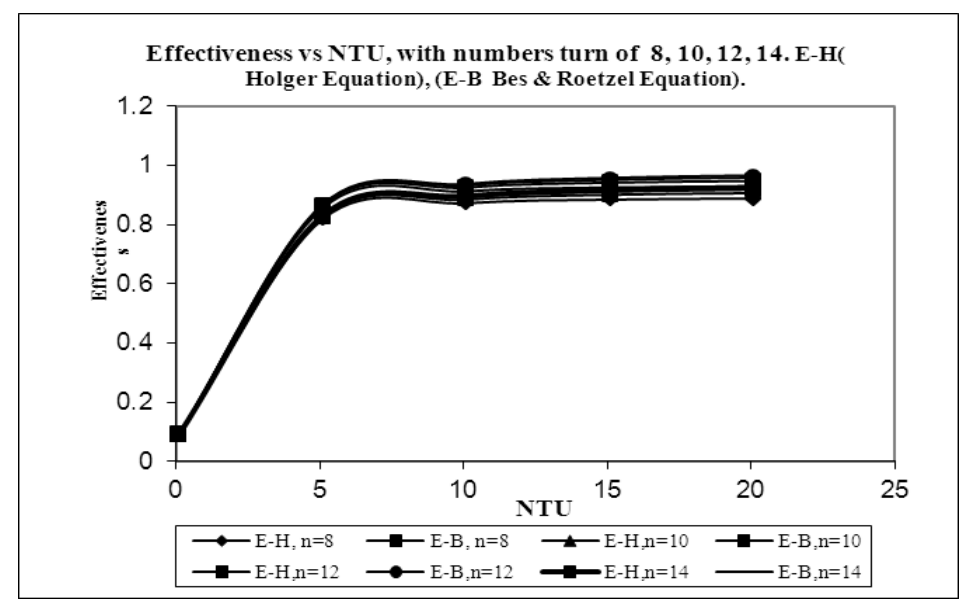

Figure 8. Effectiveness vs NTU ratio in function of numbers of turn calculated with Holger equation (E-H), and Th. Bes and W. Roetzel equation (E-B)

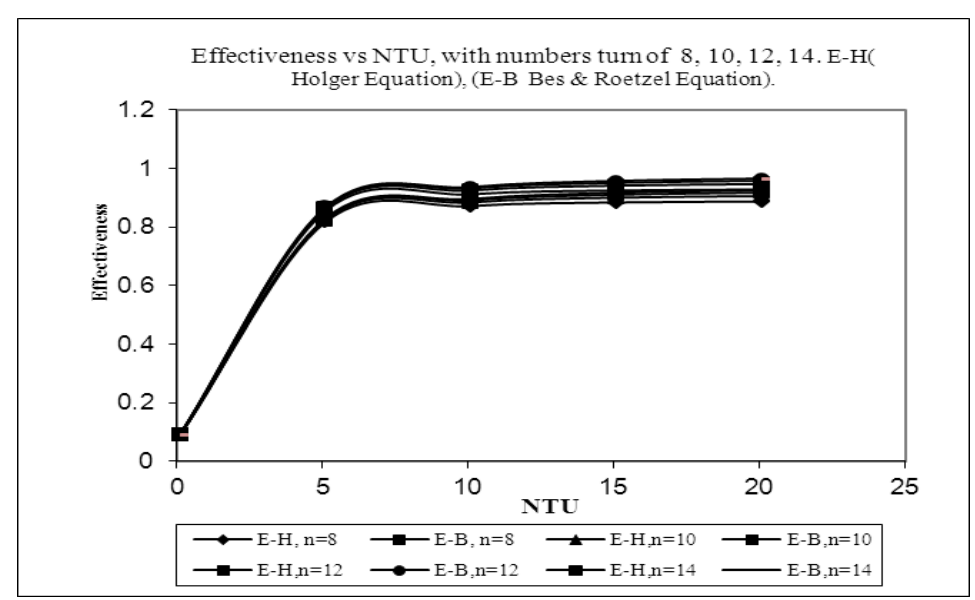

Figure 9. Effectiveness vs NTU ratio in function of numbers of turn calculated with Holger equation (E-H), and Th. Bes and W. Roetzel equation (E-B) 
Next we consider the case study II, the problem corresponds to an engine oil to be cooled by sea water from the open literature, see Table 2 . In this case we obtain $\mathrm{t}_{0}=302.997 \mathrm{~K}, \mathrm{~T}_{0}=329.885 \mathrm{~K}$ with $0.03 \%$ and $0.57 \%$ relative errors for the outlet temperatures of for the cold and hot fluid respectively and it corresponds to a SPHE with turns. As before the results are within engineering practice. The correlation between effectiveness and capacity ratio is shown in Figure 10, whereas effectiveness and NTU in Figure 11. Results are similar.

Table 2. II-Case study Specifications streams

\begin{tabular}{ccc}
\hline Item & Hot Fluid & Cold fluid \\
\hline Flow rate $(\mathrm{kg} / \mathrm{s})$ & 5 & 2.375 \\
Inlet temperature $\left({ }^{\circ} \mathrm{C}\right)$ & 65 & 20 \\
Outlet temperature $\left({ }^{\circ} \mathrm{C}\right)$ & 55 & 30 \\
Heat capacity $\left(\mathrm{J} / \mathrm{kg}{ }^{\circ} \mathrm{C}\right)$ & 1902 & 4004 \\
Thermal conductivity $\left(\mathrm{W} / \mathrm{m}{ }^{\circ} \mathrm{C}\right)$ & 0.1442 & 0.639 \\
Density $\left(\mathrm{kg} / \mathrm{m}^{3}\right)$ & 885.127 & 1013.4 \\
Pressure drop $(\mathrm{Pa})$ & 140000 & 5000 \\
Viscosity $(\mathrm{kg} / \mathrm{m} \mathrm{s})$ & $7.5 \times 10^{-3}$ & $9.640 \times 10^{-3}$ \\
Plate spacing $(\mathrm{m})$ & $4.77 \times 10^{-3}$ & $6.27 \times 10^{-3}$ \\
Plate thickness $(\mathrm{m})$ & $3.175 \times 10^{-3}$ & $3.175 \times 10^{-3}$ \\
Initial internal diameter $(\mathrm{m})$ & 0.203 & 0.203 \\
Thermal conductivity of material & 17.3 & 17.3 \\
of construction $\left(\mathrm{W} / \mathrm{m}{ }^{\circ} \mathrm{C}\right)$ & & \\
\hline
\end{tabular}

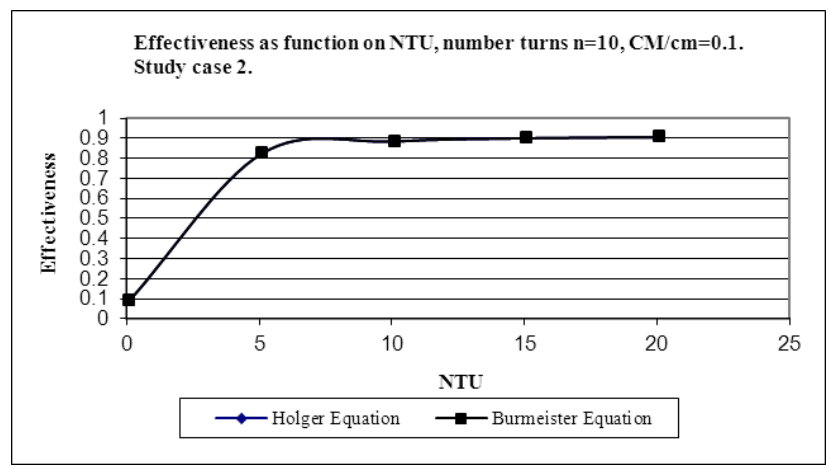

Figure 10. Efectivenessvsn(number of turns) calculated with Holger and Bes \& Roetzel equations with temperatures numerically obtained, Case Study II

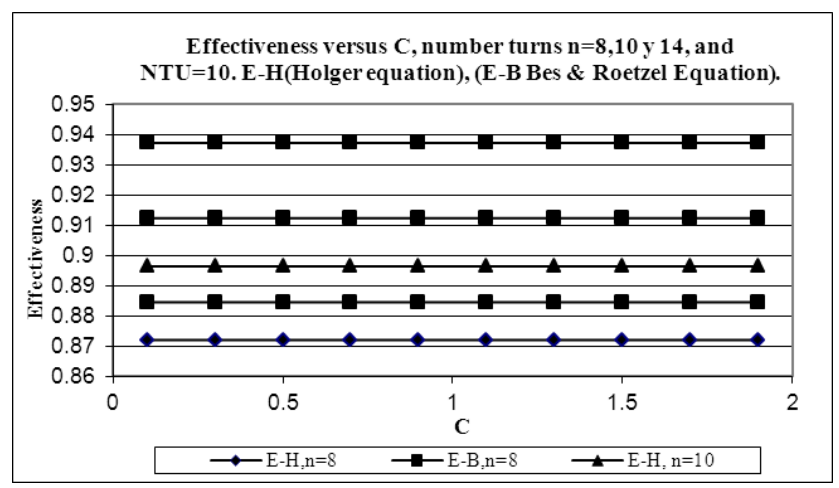

Figure 11. Effectiveness vs NTU ratio in function of numbers of turn calculated with Holger equation $(\mathrm{E}-\mathrm{H})$, and Bes \& Roetzel equation (E-B) 


\section{Conclusions}

We have proposed a numerical method to solve a system of steady state equations modeling flow in a spiral heat exchanger. The method yields the temperature profiles, and outlet temperatures. From these temperatures performance parameters are computed, LMTD correction factor and effectiveness. The numerical method is validated with experimental results from two case studies reported in the literature. The model used, equations (7)-(12), permits us to determine the overall behavior of spiral heat exchangers from temperature profiles. The underlying design method is to run several configurations and use the LMTD correction factor and effectiveness as selection criteria. The design analysis in this work is an alternative for thermal performance of a SPHE. Moreover, the parameters involved come directly from manufacturing.

At present we are interested in the following optimal design problem: Find SPHE configurations that satisfy optimality conditions in terms of effectiveness, or correction factor, as the objective functions. We shall to report on this elsewhere.

\section{Acknowledgements}

The authors wish to thank the referees for their suggestions which have improved the manuscript greatly.

\section{References}

Bes, Th., \& Roetzel, W. (1992). Distribution of heat flux density in spiral heat exchangers. Int. J. Heat Mass Transfer, 35(6), 1331-1347. http://dx.doi.org/10.1016/0017-9310(92)90026-O

Bounopane, R. A., \& Troupe, R. A. (1970). Analytical and experimental studies in a spiral heat exchangers. Fourth Proc. Int: Heat Transfer Conf., 1-11. http://dx.doi.org/10.1115/1.1857950

Choudhury, K., Linkmeyer, H., Bassiouny, K., \& Martin, H. (1985). Analytical studies on the temperature distribution in a spiral plate heat exchangers. Straightforward Design formulae for efficiency and mean temperature difference. Chem. Eng. Process, 19, 183-190. http://dx.doi.org/10.1016/0255-2701(84)80020-3

Cieslinski, P. J., \& Bes, T. (1983). Analytical Heat transfer studies in a spiral plate heat exchangers Proc. XV Ith Int. Congr. Refrigeration, 449-454.

Egner, M. W, \& Burmeister, L. C. (2005). Heat Transfer for Laminar Flow in Spiral Ducts of Rectangular Cross Section. Transactions of the ASME, 127. http://dx. doi.org/10.1115/1.1857950

Hupkes, H. J., \& Augeraud-Veron, E. (2011). Well-Posedness of initial value problems for functional differential and algebraic equations of mixed type. Discrete and Continuous Dynamical Systems, 30(3), 737-785. http://dx.doi.org/10.3934/dcds.2011.30.737

Holger, M. (1992). Heat exchangers. London: Hemisphere Publishing Corporation.

Minton, P. (1971). Designing spiral plate heat exchangers. Chem. Eng. Prog., 77, 103-112.

Morimoto, E., \& Hotta, K. (1988). Study of the Geometric Structure and Heat Transfer Characteristic of a Spiral Plate Heat Exchanger. Heat Transfer, Japanese Research, 17, 53-71.

Saravanan, K., \& Rajavael, R. (2008). An Experimental Investigation of Heat Transfer Coefficients for Spiral Plate Heat Exchanger. Modern Applied Science, 2(5), 14-20.

Strelow, O. (2000). A general calculation method for plate heat exchangers. Int. J. Therm. Sci., 39, 645-658. http://dx.doi.org/10.1016/S1290-0729(00)00228-3

Strenger, M. R., Churchill, S. W., \& Retallick, W. B. (1990). Characteristics of a double-spiral Heat Exchanger for the Catalytic Incineration of Contaminated Air. Ind. Eng. Chem. Res., 29, 1997-1984. http://dx.doi.org/10.1021/ie00105a033

Wilhelmsson, B. (2005). Consider spiral heat exchangers for fouling application. Hydrocarbon Processing, July, 81-83.

Zaleski, T., \& Lachowski, A. (1984). Unsteady temperature profiles in parallel flow spiral heat exchangers. Int. Chem. Eng., 27, 556-565. http://dx.doi.org/10.1016/S1359-4311(99)00028-9 